\title{
La predicción de series temporales mediante el modelo de regresión
}

(Time series prediction using the regression model)

\author{
Dr. Agustín ALONSO RODRIGUEZ \\ Real Centro Universitario \\ "Escorial-María Cristina" \\ San Lorenzo del Escorial
}

\begin{abstract}
Resumen: En este trabajo se expone cómo la predicción en las series temporales se puede obtener acudiendo al modelo de regresión. Utilizando la serie Indice de la Red Eléctrica de España, REE, en su apartado: demanda eléctrica para la construción de edificios, desde enero de 2010 hasta agosto del 2020, datos corregidos, se muestra paso a paso cómo lograrlo.
\end{abstract}

Abstract: In this paper, it is shown how the prediction of a time series can be achieved using the regression model. The time series Indice of Red Eléctrica de España, REE, section: demand of electricity for buildings construction, from January 2020 to August 2020, it is used as an illustration.

Palabras clave: Serie Indice, demanda de electricidad, construcción de edificios, Red Eléctrica de España, modelo de regresión, predicción, series temporales, paquetes estadísticos: forecast, R, TSstudio.

Keywords: Time series: Indice of Red Electríca de España, demand of electricity, buildings construction, regression model, time series prediction, software: forecast, R, TSstudio.

\section{Sumario:}

\section{Introducción.}

II. La Demanda de energía electrica para la construcción de edificios, la serie Índice de REE.

III. El estudio de las series de tiempo. 
IV. Modelos de regresión para la serie Demanda de energía eléctrica.

V. Modelos validados y ajuste de sus predicciones.

VI. Un modelo ARIMA.

VII. Conclusiones.

VIII. Bibliogafía.

Recibido: noviembre 2020.

Aceptado: enero 2021.\# 


\section{INTRODUCCIÓN}

La descomposición de una serie temporal en sus elementos estructurales: la tendencia, la estacionalidad, el ciclo, y el componente irregular, permiten incorporar, en el marco del modelo de regresión, el análisis de las series temporales.

En la descomposición de una serie temporal en sus elementos estructurales, cabe distinguir una doble estructura, a saber: aditiva y multiplicativa.

Si designamos por $Y_{t}$ la serie temporal, podemos escribir la estructura aditiva como

$$
Y_{t}=T_{t}+S_{t}+C_{t}+I_{t}
$$

siendo:

$T_{t}:$ la tendencia, trend en inglés

$S_{t}$ : el componente estacional, season, relacionado con la frecuencia de la serie: mensual, trimestral, etc.

$C_{t}$ : el componente cíclico

$I_{i}$ : el componente irregular.

Y por lo que respecta a la estructura multiplicativa tenemos

$$
Y_{t}=T_{t} \times S_{t} \times C_{t} \times I_{t}
$$

siendo el significado de los símbolos como en el caso anterior.

La estructura aditiva en (1), permite asociarla con el modelo de regresión lineal al escribir, sin tener en cuenta el componente cíclico, incorporado al elemento estacional

$$
Y_{t}=\beta_{1}+\beta_{2} T_{t}+\beta_{3} S_{t}+\varepsilon_{t}
$$


siendo ahora

$Y_{t}$ : la variable respuesta, y $T_{t}$ y $S_{t}$ las variables explicativas, asociando el término irregular con el término de error del modelo de regresión: $\varepsilon_{t}$.

Por lo que respecta a la estructura multiplicativa en (2), mediante la transformación logarítmica de sus componentes, podemos hacerla aditiva escribiendo

$$
\log \left(Y_{t}\right)=\beta_{1}+\beta_{2} \log \left(T_{t}\right)+\beta_{3} \log \left(S_{t}\right)+\varepsilon_{t}
$$

Advertencia. Dada la amplitud del tema y las restricciones de espacio de toda publicación, para facilitar la comprensión, se ha optado por las representaciones gráficas a costa de las explicaciones pormenorizadas de los resultados. De esta manera, espero haber logrado el objetivo fijado en el título del artículo.

\section{LA SERIE ÍNDICE DE RED ELÉCTRICA DE ESPAÑA.}

A fin de mostrar el lado aplicado de este enfoque para el análisis de las series temporales, utilizaré la serie Índice de la Red Eléctrica de España, en su apartado: demanda de energía eléctrica para la construcción de edificios, una serie mensual (datos corregidos) de 128 observaciones, desde enero del 2010 hasta agosto del 2020 (datos accedidos el 12-09-2020). Con el nombre demanda la serie aparece representada en la figura 1.

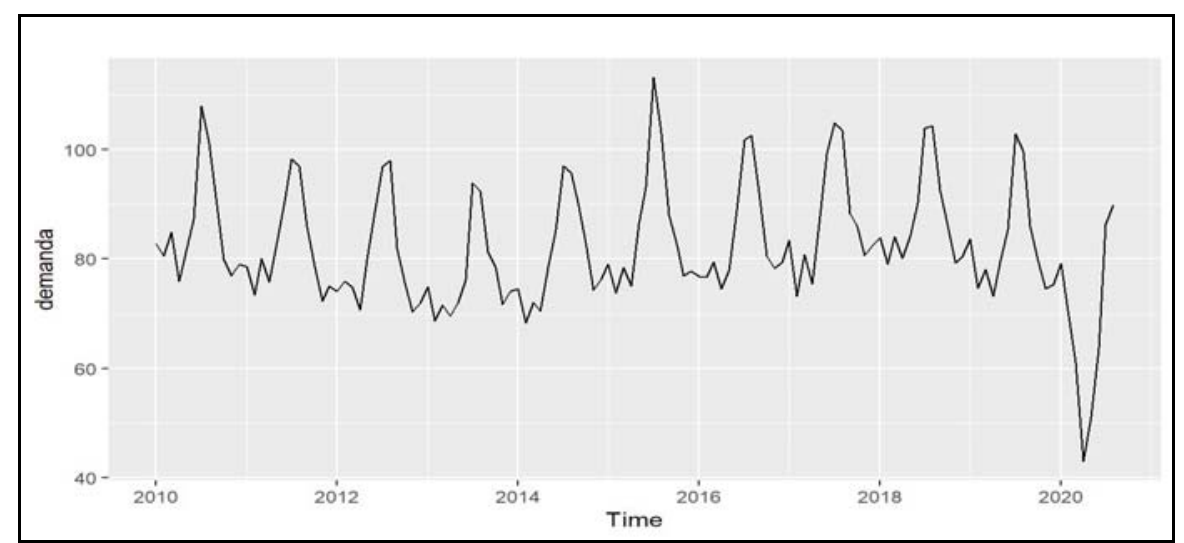

Figura 1. Demanda de electricidad para la construcción de edificios. 


\section{EL ESTUDIO DE LAS SERIES DE TIEMPO}

A lo largo de los años, el análisis de las series temporales se ha llevado a cabo tanto en el ámbito de la frecuencia, como en el ámbito del tiempo. En este último ámbito, y desde la aparición en 1976 de la obra de Box y Jenkins, Time Series Analysis: Forecasting and Control, el análisis de las series de tiempo se ha llevado a cabo, preferentemente, sobre la base de la información contenida en la función de autocorrelación y de autocorrelación parcial de la serie en estudio: los modelos ARIMA.

Gracias a Rob. J. Hyndman y colaboradoes, 2008, el paquete forecast, parte del sistema estadístico fpp (Forecasting: Principles and Practice) permite, gracias su función tslm aplicar el análisis de las series temporales en el marco de la regresión. Así mismo, hay que destacar los trabajos de Rami Krispin, quien en 2019, publica Hands-on Time Series Analysis with $R$, acompañado por su paquete de software TSstudio. Más recientemente R. Krispin ha presentado el software forecast $L M$, todavía en desarrollo, dedicado todo él al análisis de las series temporales en el marco de la regresión.

Tanto Hyndman como Krispin presentan todo un sistema de software para el análisis de las series temporales, con excelentes capacidades gráficas. La diferencia fundamental es que forecast utiliza ggplot2 para las representaciones gráficas, con su facil adaptación al formato PDF para publicaciones. Por su parte, Krispin utiliza Plotly para sus gráficos. Destacar también que Krispin posibilita la incorporación de forecast a su software, permitiendo que algunas funciones de ambos sistemas funcionen conjuntamente.

\section{MODELOS DE REGRESIÓN PARA LA SERIE DEMANDA DE ENERGÍA ELÉCTRICA}

Comenzamos nuestro análisis particionando la serie demanda en dos subseries: la subserie train, sobre la que se estima el modelo, y la subserie test que permite obtener estadísticos sobre lo acertado del ajuste. Por tratarse de datos mensuales, la subserie test comprende las 12 últimas observaciones, todo un año, figura 2.

Hecha esta partición, vamos a estimar con la función $t s l m$ tres modelos para nuestra serie: un modelo para la tendencia, un modelo para el componente estacional y un tercer modelo, incorporando ambos componentes. 


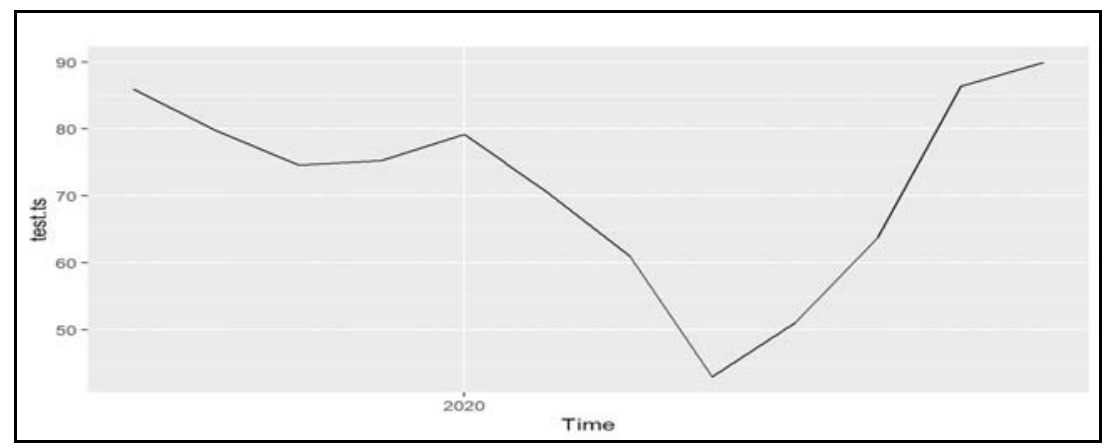

Figura 2. Las 12 observaciones de la subserie test.

\section{Modelo para la tendencia: modelo1}

El modelo a estimar es:

$$
Y_{t}=\beta_{1}+\beta_{2} \text { trend }_{t}+\varepsilon_{t}
$$

obteniendo como resultado

$$
\begin{gathered}
\hat{Y}_{t}=80.303+0.053 \text { trend }_{t} \\
(1.856))(0.028) \\
R^{2}=0.031
\end{gathered}
$$

(entre paréntesis los errores estándar de los coeficientes)

Podemos representar el ajuste de este modelo junto a los datos muestrales como se muestra en la figura 3 .

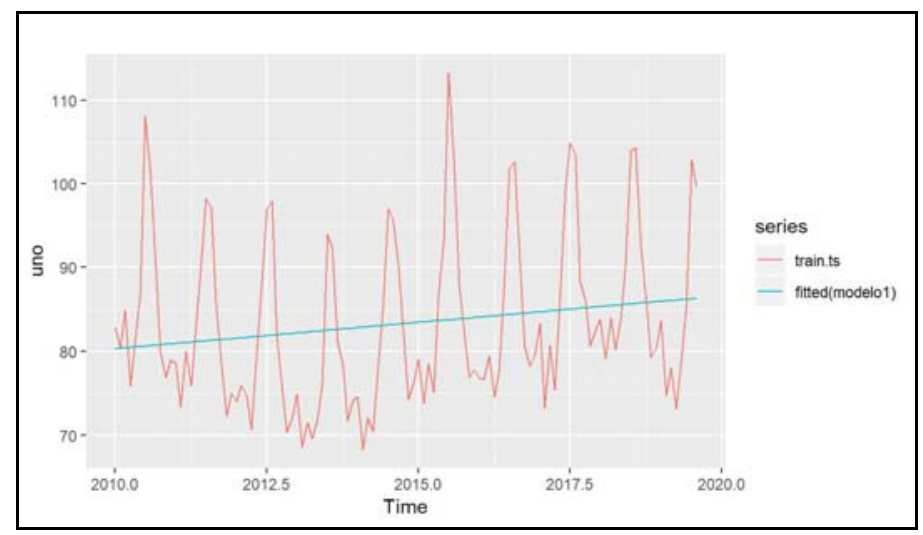

Figura 3. Datos muestrales y ajuste del modelo1, 


\section{Modelo para el comportamiento estacional: modelo2}

El modelo a estimar es:

$$
Y_{t}=\beta_{1}+\beta_{2} \text { season }_{t}+\varepsilon_{t}
$$

con el resultado:

\# Call:

\# tslm(formula = train.ts season)

\#

\# Residuals:

$\begin{array}{rrrrrr}\# & \text { Min } & 1 Q & \text { Median } & 3 Q & \text { Max } \\ \# & -12.3464 & -3.0134 & 0.3247 & 2.6897 & 11.1845\end{array}$

\#

\# Coefficients:

\#

\#

\# Intercept

Estimate Std.

Error $t$ value

$1.363 \quad 58.111$

$\operatorname{Pr}(>|\mathrm{t}|)$

\# season2

79.193

1.927

$-2.468$

$<2 \mathrm{e}-16$ ***

\# season3

$-4.756$

1. 927

$-0.404$

๑. 01523 *

\# season4

$-0.779$

1. 927

$-2.649$

$\odot .68690$

\# season5

1. 700

1. 927

๑. 882

9.310

1. 927

4.831

$\odot .00932$ **

\# season6

22.901

1. 927

11.882

๑. 37991

\# season 7

20.547

1. 927

10.661

4. $70 \mathrm{e}-06 * * *$

\# season8

8.636

1. 980

4. 362

$<2 \mathrm{e}-16 * * *$

\# season 9

2.042

1.980

1. 031

1.980

$-1.794$

$-3.552$

1.980

$-0.908$

$<2 \mathrm{e}-16$

3. $05 \mathrm{e}-05 * * *$

๑. 30473

0.07571

$-1.798$

$\odot .36604$

\#---

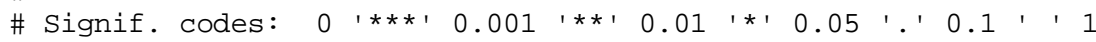
\#

\# Residual standard error: 4.309 on 104 degrees of freedom

\# Multiple R-squared: 0.8335 , Adjusted R-squared: 0.8159

\# F-statistic: 47.33 on 11 and 104 DF, p-value: < 2.2e-16

Podemos también representar los datos muestrales junto al ajuste de este modelo como se muestra en la figura 4

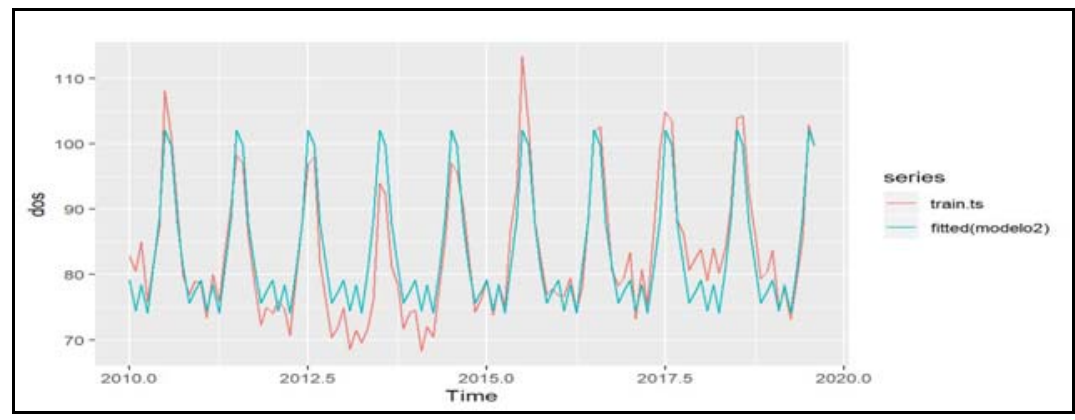

Figura 4. Datos muestrales y ajuste del modelo2. 


\section{Modelo completo: modelo3}

En este modelo incorporamos ambos componentes: la tendencia y el componente estacional:

$$
Y_{t}=\beta_{1}+\beta_{2} \text { trend }_{t}+\beta_{3} \text { season }_{t}+\varepsilon_{t}
$$

obteniendo como resultado:

\#

\# Call:

\# tslm(formula = train.ts $\sim$ trend + season)

\#

\# Residuals:

\# $\quad$ Min $1 Q$ Median $3 Q \quad$ Max

$\begin{array}{llllll}\# & -11.5937 & -2.6815 & 0.0601 & 2.8104 & 10.9336\end{array}$

\#

\# Coefficients:

\# Estimate Std.

\# (Intercept) 76.89309

\# trend

76.89309
0.04181

Error $t$ value

1.42883

53.815

๑. 01130

3.700

$-4.79751$

1.81949

$-2.637$

$1.81959-0.474$

\# season3

$-0.86263$

1.81977

$-2.875$

1.82002

0.842

1.53224

1.82033

9.10143

1.82072

5.000

$\operatorname{Pr}(>|\mathrm{t}|)$

$<2 \mathrm{e}-16$

$\odot .000348$

$\odot .009666$ *

$\odot .636448$

0.004911 * *

\# season5

22.64971

1.82117

20.25460

12.440

๑ . 401804

\# season 7

8.55280

1.86945

11.122

2. 36 e- 06

$<2 e-16$

\# season 9

1. 91688

1. 86962

4.575

$<2 \mathrm{e}-16 * * *$

\# season10

$-3.71960$

1.86986

1. 025

1. 33 e- 05

\# season11

$-2.00675$

1. 87017

$-1.989$

0.307634

\# season12

\# ---

\# Signif. codes:

$01 * * * 10.001$

$1 * * 10.01$

$-1.073$

$\odot .049326 *$

$\odot .285763$ \#

\# Residual standard error: 4.068 on 103 degrees of freedom

\# Multiple R-squared: $0.853, \quad$ Adjusted R-squared: 0.8359

\# F-statistic: 49.82 on 12 and 103 DF, p-value: < 2.2e-16

Los datos muestrales junto al ajuste de este modelo aparecen en la figura 5.

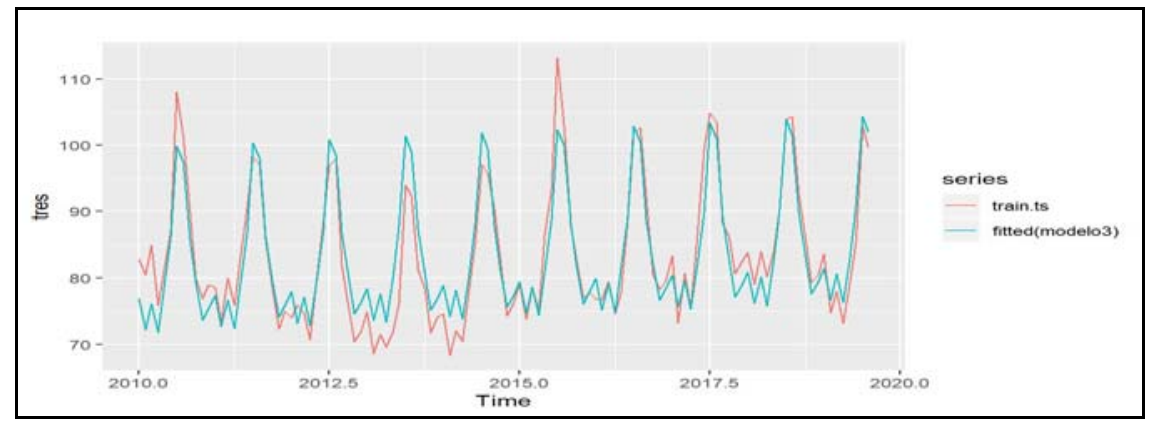

Figura 5. Datos muestrales y ajuste del modelo3 
Llegados aquí, se impone comprobar la validez de este modelo3, el más completo. Analizando los residuos, tenemos, la figura 6.

\section{Residuals from Linear regression model}

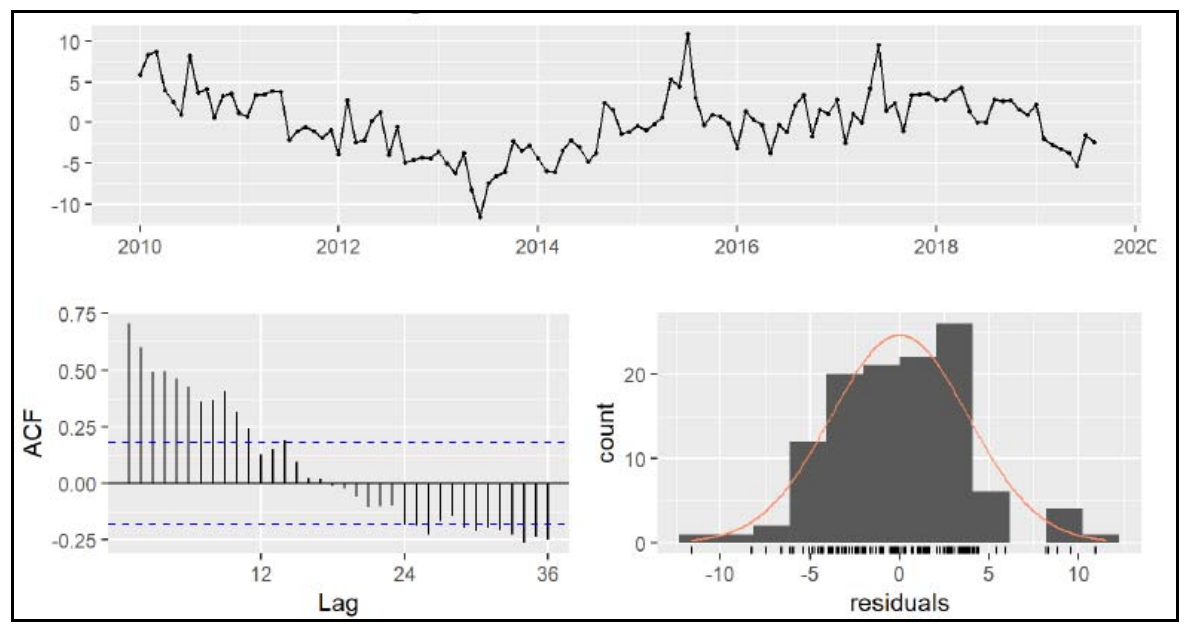

Figura 6. Residuos del modelo3.

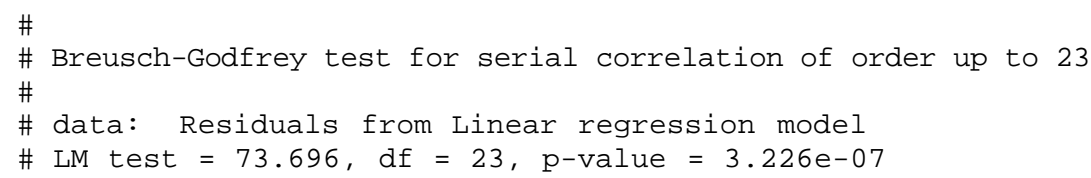

La alta autorrelación presente en estos residuos invalida, estadísticamente, el modelo estimado.

Para corregir este problema caben dos opciones. La primera consiste en modificar el modelo introduciendo retardos, lags, como regresores. La segunda consiste en modelizar los residuos, utilizando las facilidades que brinda el paquete forecast, acudiendo a la función auto.arima. Veamos ambas posibilidades.

\section{MODELOS VALIDADOS Y AJUSTES DE SUS PREDICCIONES}

\section{Retardos como regresores de un modelo}

Como guía para establecer el número de retardos a tener presente, estimemos la función de autocorrelación parcial de los residuos: figura 7. 


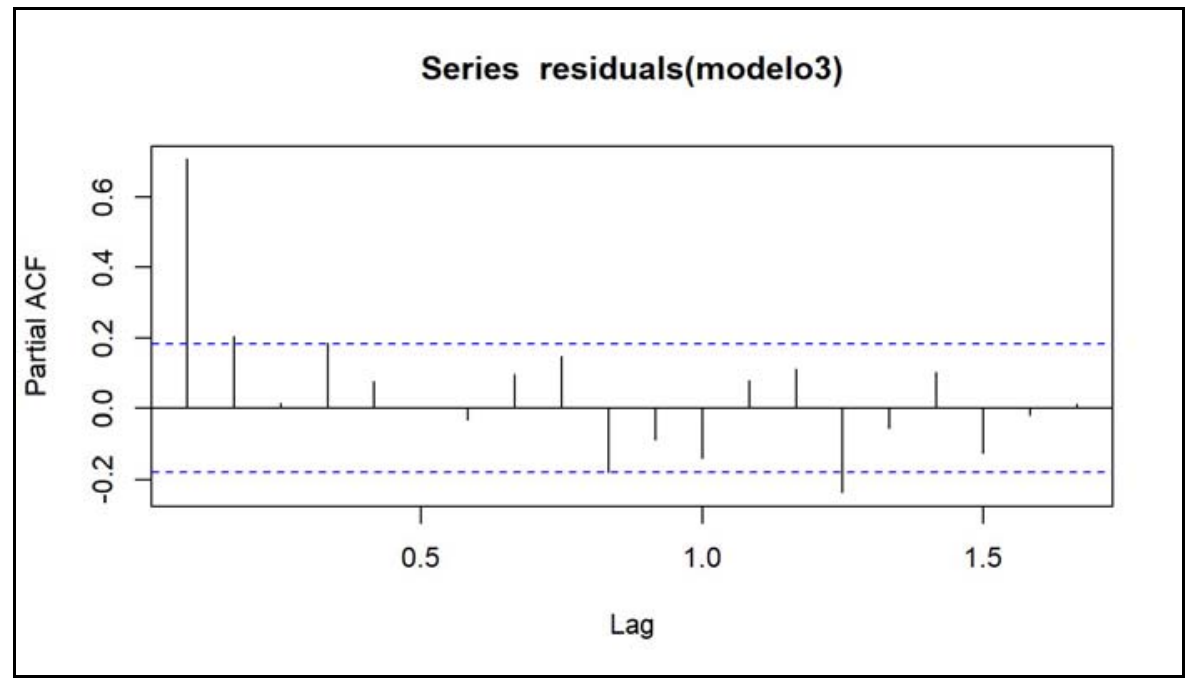

Figura 7. Función de autocorrelación parcial de los residuos del modelo3.

A la vista de este resultado, podemos considerar, inicialmente, un modelo $A R(1)$ para los residuos, y postular un retardo: lag1, como posible regresor de un nuevo modelo.

Siguiendo las sugerencias de R. Krispin, y con ayuda de su paquete TSstudio formamos una tabla o data.frame con los datos de la serie demanda, en la que incorporamos la fecha de la serie, date, la serie, $y$, el retardo, lagl y los meses, month. Como resultado, tenemos

\begin{tabular}{|c|c|c|c|c|}
\hline \# & date & $\mathrm{y}$ & lag1 & month \\
\hline \# 1 & $2010-01-01$ & 82.833 & NA & ene \\
. \\
\hline \# 2 & $201 \odot-\odot 2-\odot 1$ & 80.522 & 82.833 & $b \backslash$ \\
\hline \# 3 & $2010-03-01$ & 84.960 & 80.522 & \\
\hline \# 4 & $2010-\odot 4-01$ & 75.876 & 84.960 & \\
\hline \# 5 & $2010-05-01$ & 81.247 & 75.876 & \\
\hline \# 6 & $2010-\odot 6-\odot 1$ & 87.239 & 81.247 & $j u n 18$ \\
\hline
\end{tabular}

Hecha esta reorganización de la serie original, volvemos a realizar la partición, como antes, en dos subseries: train y test

Tras las particiones, estimamos el nuevo modelo, $\bmod 1, \mathrm{y}$ comprobamos sus residuos, figura 8 .

$$
Y_{t}=\beta_{1}+\beta_{2} \text { trend }_{t}+\beta_{3} \text { season }_{t}+\beta_{4} \operatorname{lag} 1_{t}+\varepsilon_{t}
$$


Residuals from Linear regression model

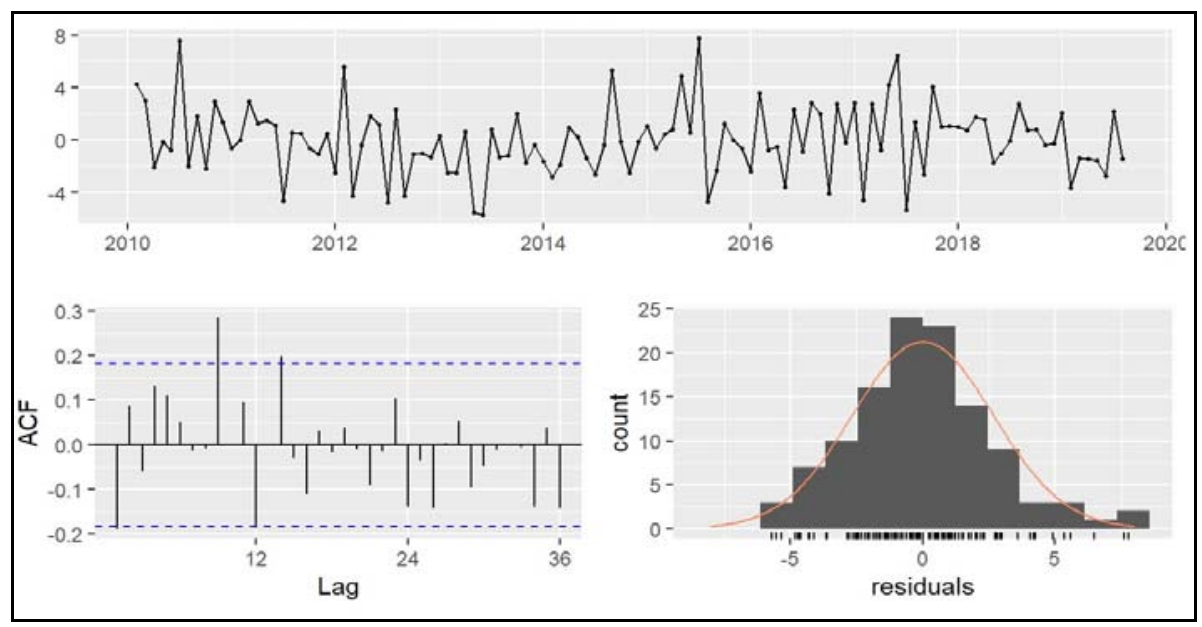

Figura 8: Residuos del modelo mod1.

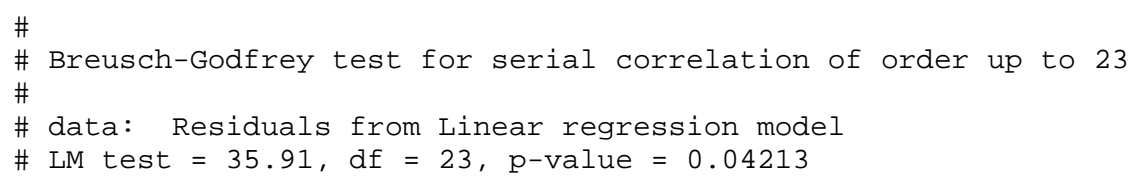

Ahora es posible aceptar que los residuos validan el modelo modl

Validado el modelo, podemos pasar a la predicción puntual con el mismo, obteniendo, como resultado, la siguiente tabla, y su representación en la figura 9.

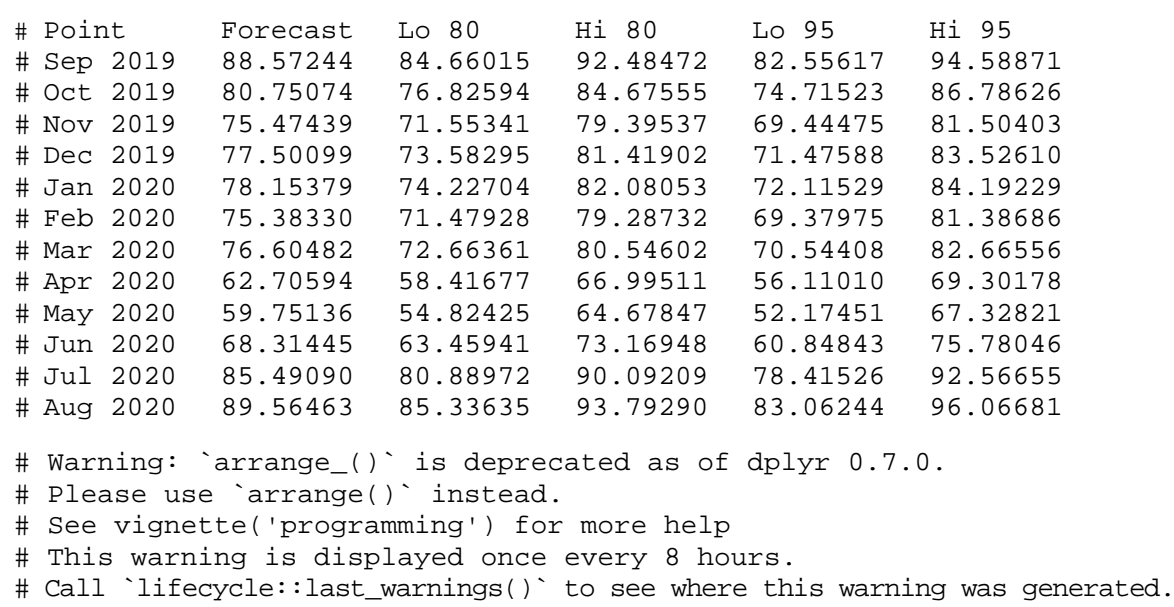




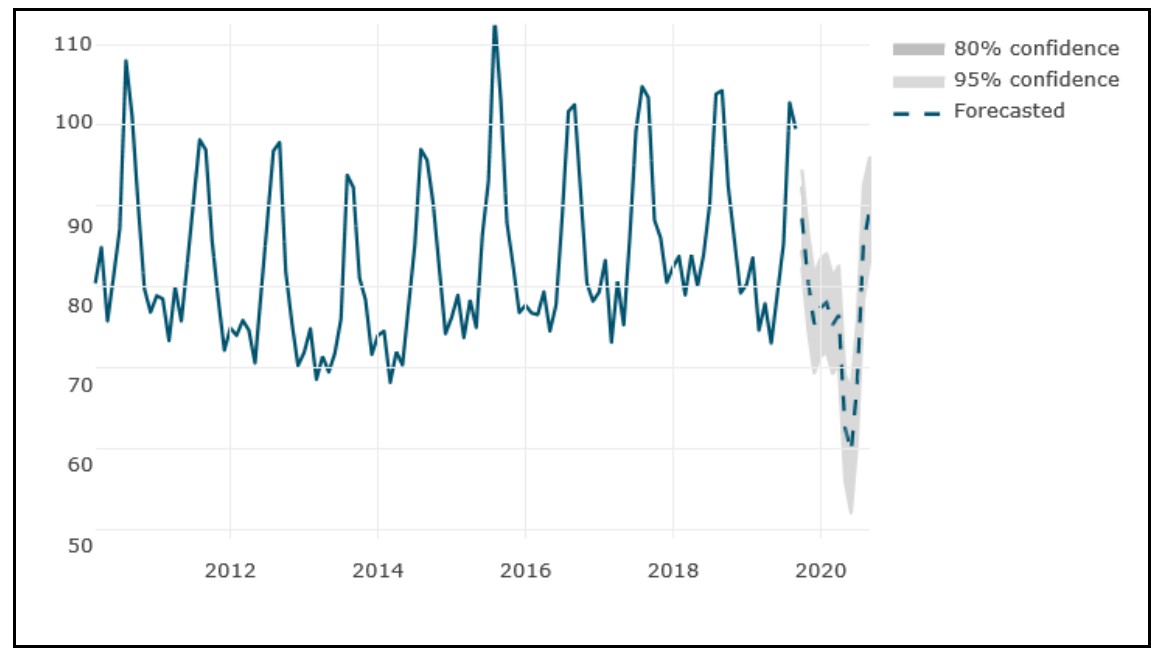

Figura 9. Predicciones con el modelo modl.

Con ayuda de TSstudio, es posible representar, con mayor detalle, los datos de la serie completa, los datos de la predicción, junto a los datos de la submuestra test. El problema es que al imprimirse el gráfico en blanco y negro, el resultado queda poco realzado, véase la figura 10.

\section{demanda - Actual vs Forecasted and Fitted}

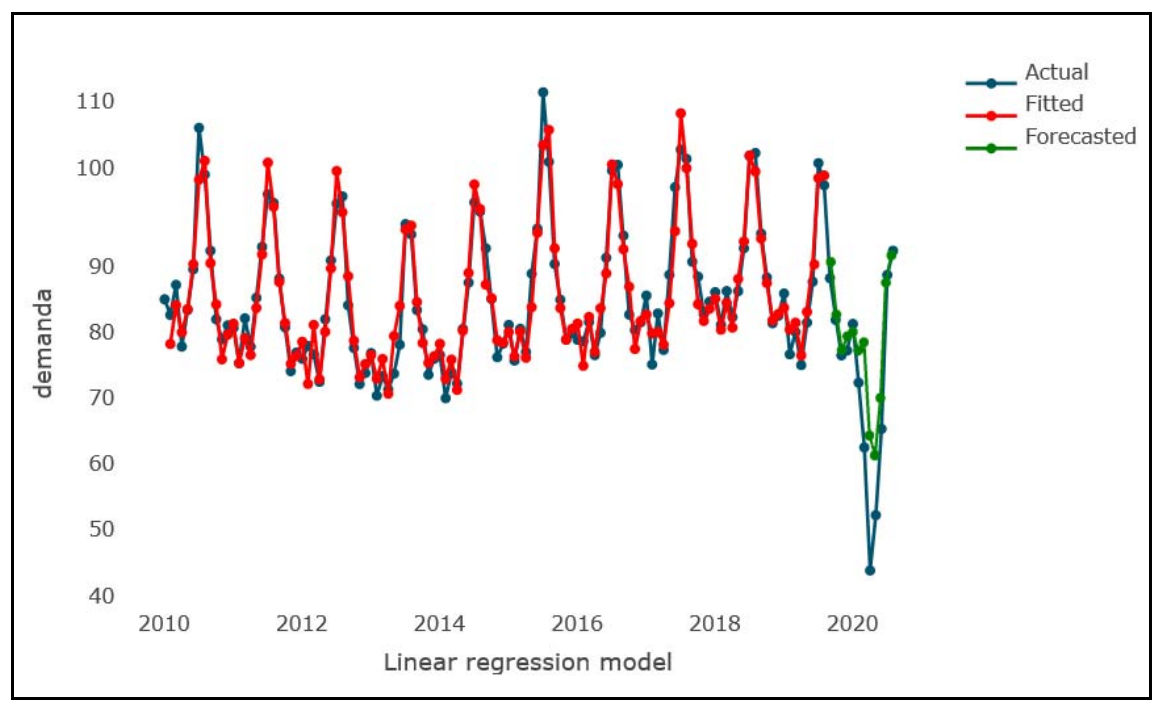

Figura 10. Datos muestrales, predicciones fc1 y submuestra test. 


\section{Modelización de los residuos mediante auto.arima}

El segundo enfoque para eliminar la alta autocorrelación presente en los residuos consiste en establecer un modelo para los mismos.

Hyndman y Athanasopoulos en el capítulo 9 de su Forecasting, Principles and Practice, segunda edición, 2018, al tratar los modelos de regresión dinámicos, en el apartado segundo, 9.2, considera el tema Regression with ARIMA errors in $R$, que permite estimar un modelo de regresión con residuos que, a su vez, son modelizados siguiendo un modelo ARIMA. Cf. p. 279.

En esta ocasión, siguendo a Rami Krispin, pp. 382 - 386, en su adaptación de lo expuesto por Hyndman y Athanasopoulos, se estima el modelo mod2.

Tras unos minutos, obtenemos el modelo estimado

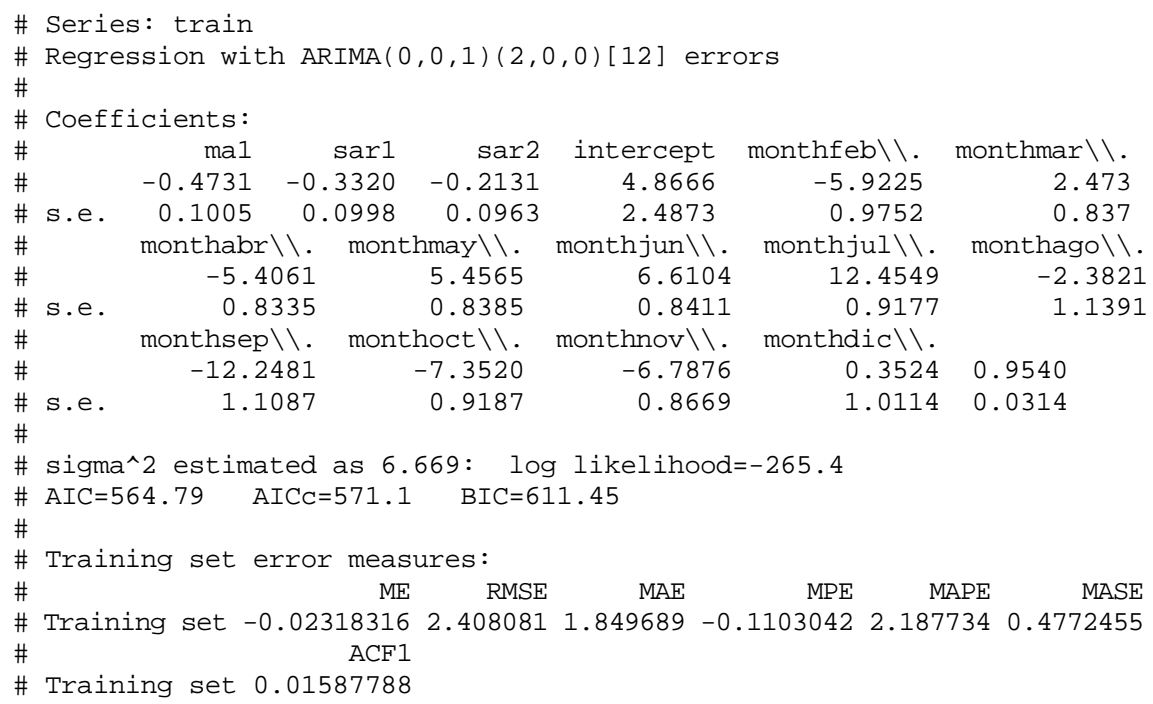

Los residuos de este modelo, han sido modelizados, de manera automática, como ARIMA $(0,0,1)(2,0,0)$ [12], y una vez modelizados, los residuos del modelo $\bmod 2$ aparecen en la figura 11.

Residuals from Regression with ARIMA $(0,0,1)(2,0,0)[12]$ errors.

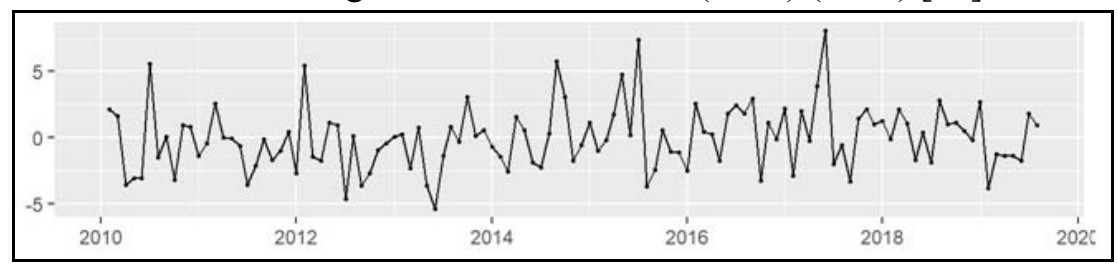




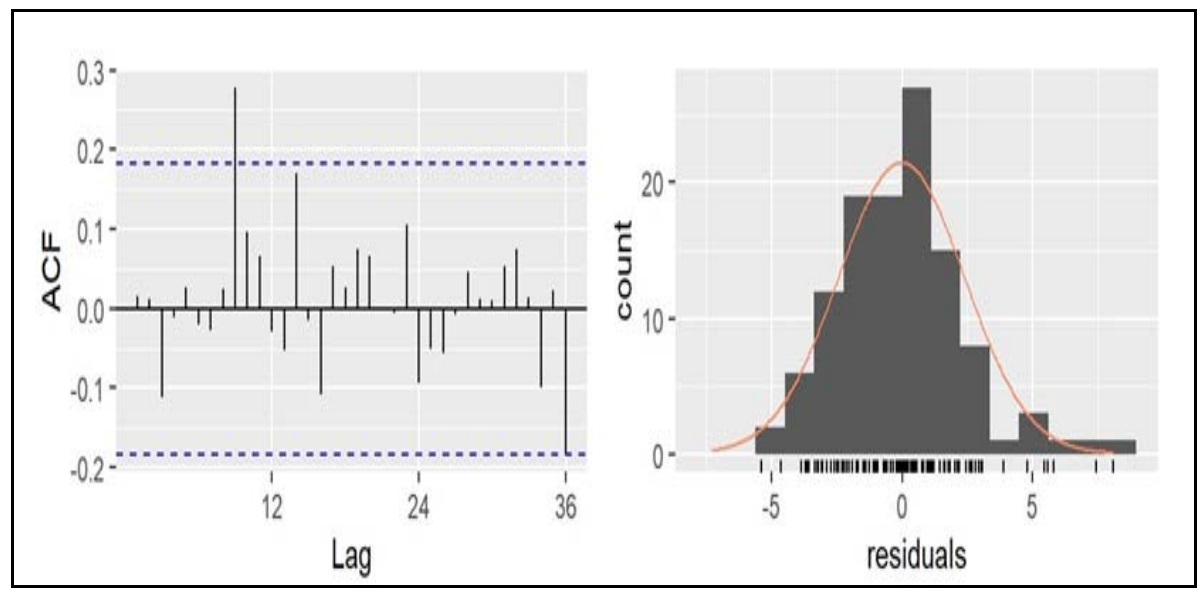

Figura 11. Residuos del modelo $\bmod 2$.

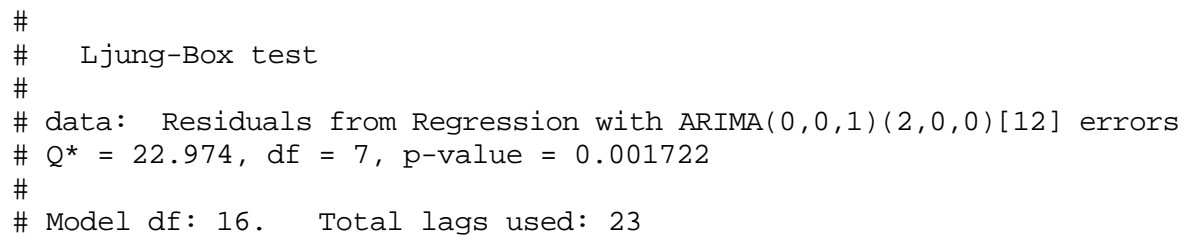

Como puede observarse, los residuos validan el modelo mod2.

Validado el modelo, se pasa a la predicción con el mismo. Sus valores aparecen en la siguiente tabla.

$\begin{array}{llllll}\text { \# Point } & \text { Forecast } & \text { Lo } 80 & \text { Hi } 80 & \text { Lo } 95 & \text { Hi } 95 \\ \text { \# Sep 2019 } & 87.71992 & 84.41046 & 91.02937 & 82.65855 & 92.78129 \\ \text { \# Oct 2019 } & 78.48729 & 74.82616 & 82.14841 & 72.88808 & 84.08649 \\ \text { \# Nov 2019 } & 74.41469 & 70.75356 & 78.07581 & 68.81548 & 80.01390 \\ \text { \# Dec 2019 } & 76.46612 & 72.80499 & 80.12724 & 70.86691 & 82.06533 \\ \text { \# Jan 2020 } & 75.93447 & 72.27334 & 79.59559 & 70.33526 & 81.53368 \\ \text { \# Feb 2020 } & 75.82794 & 72.16681 & 79.48906 & 70.22873 & 81.42714 \\ \text { \# Mar 2020 } & 74.59486 & 70.93373 & 78.25598 & 68.99565 & 80.19407 \\ \text { \# Apr 2020 } & 57.81758 & 54.15645 & 61.47870 & 52.21837 & 63.41679 \\ \text { \# May 2020 } & 52.11895 & 48.45782 & 55.78007 & 46.51974 & 57.71816 \\ \text { \# Jun 2020 } & 61.09431 & 57.43318 & 64.75543 & 55.49510 & 66.69351 \\ \text { \# Jul 2020 } & 76.71646 & 73.05533 & 80.37758 & 71.11725 & 82.31567 \\ \text { \# Aug 2020 } & 84.68098 & 81.01986 & 88.34211 & 79.08177 & 90.28019\end{array}$


La representación gráfica se recoge en la figura 12.

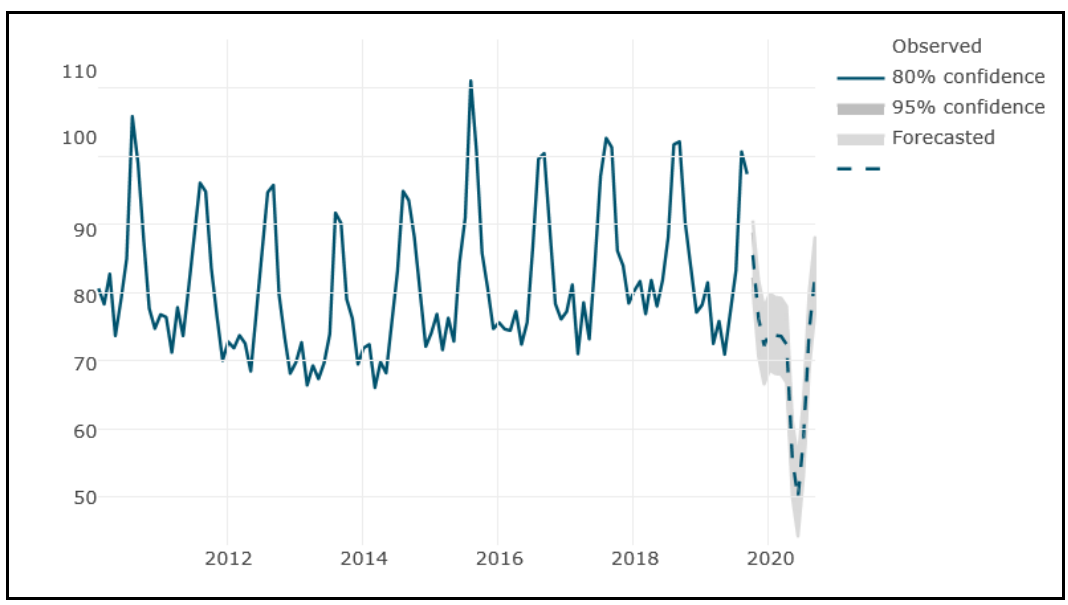

Figura 12. Predicción con el modelo mod2.

\section{Ajustes de sus predicciones}

Podemos comparar las predicciones de ambos modelos acudiendo a los estadísticos de ajuste. Para el modl tenemos,

$$
\operatorname{accuracy}\left(f_{c} 1, \text { test }\right)
$$

que da como resultado

$\begin{array}{llllll}\text { \# } & \text { ME } & \text { RMSE } & \text { MAE } & \text { MPE } & \text { MAPE } \\ \text { \# Training set } & 1.112325 e-15 & 2.657333 & 2.054429 & -0.09860793 & 2.436091 \\ \text { \# Test set } & -4.803312 \mathrm{e}+00 & 7.998320 & 5.202259 & -8.99698601 & 9.473989 \\ \text { \# } & \text { MASE } & \text { ACF1 } & \text { Theil's U } & \\ \text { \# Training set } & 0.5305327 & -0.1881583 & \text { NA } & \\ \text { \# Test set } & 1.3434240 & 0.6075475 & 0.7636603 & & \end{array}$

Para el $\bmod 2$,

$$
\operatorname{accuracy}(f c 2, \text { test })
$$

obteniendo

$\begin{array}{lclllll}\text { \# } & \text { ME } & \text { RMSE } & \text { MAE } & \text { MPE } & \text { MAPE } & \text { MASE } \\ \text { \# Training set }-\odot .02318316 & 2.408081 & 1.849689 & -0.1103042 & 2.187734 & 0.4772455 \\ \text { \# Test set } & -1.27046198 & 6.953342 & 5.007931 & -3.5538501 & 8.104801 & 1.2921159 \\ \text { \# } & \text { ACF1 } & \text { Theil's U } & & & \\ \text { \# Training set } & 0.01587788 & \text { NA } & & & \\ \text { \# Test set } & 0.56005863 & 0.6122006 & & & \end{array}$


Para la explicaciones de todos estos estadísticos, ver Hyndman y Athanasopoulos, Forecasting: Principles and Practice, segunda edición, pp. $62-68$.

Tomado en consideración el estadístico MAPE (Mean absolute percentage error), podemos ver que el porcentaje de error del primer modelo es del $2.4 \%$ en la submuestra train y del $9.5 \%$ en la submuestra test. Y para el segundo modelo, el porcentaje de error es del $2.2 \%$ en la submuestra train y del $8.1 \%$ en la submuestra test. Las diferencias son prácticamente insignificantes, quedando así aceptados ambos modelos.

\section{UN MODELO ARIMA}

Como complemento de todo lo expuesto, y con ayuda de la función auto.arima del paquete forecast podemos estimar un modelo de series temporales para nuestra serie demanda.

Una vez particionada la serie en las submestras train.ts y test.ts, como anteriormente, estimamos el modelo mediante la instrucción.

$$
\bmod 3=\text { auto. } \text { arima(train. ts })
$$

obteniendo

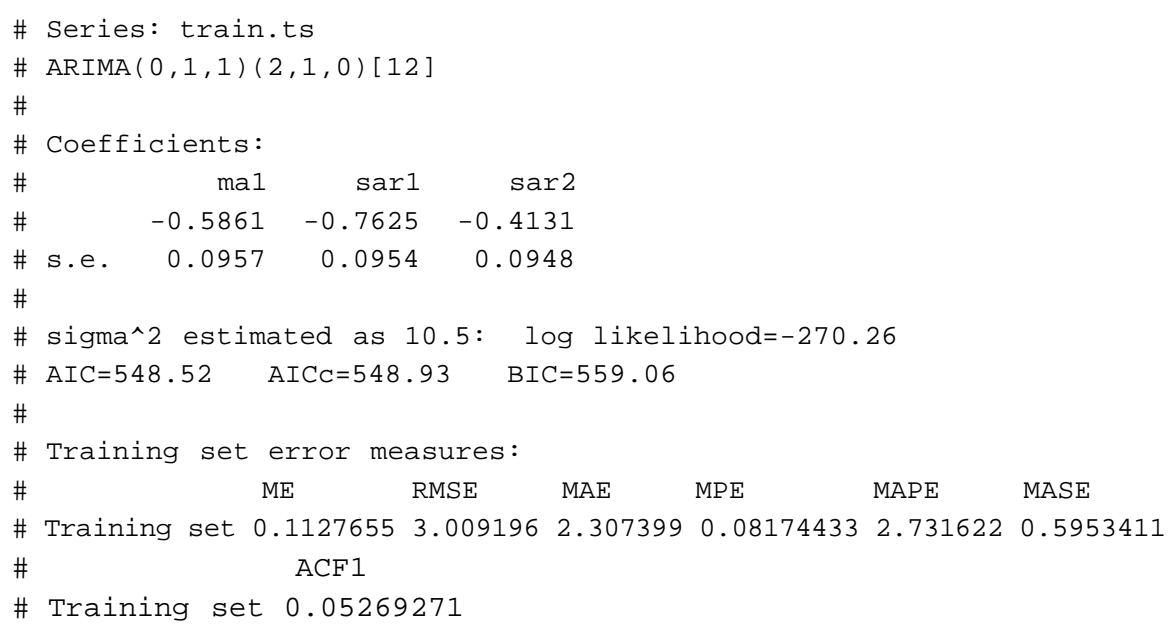


Los residuos de este modelo aparecen en la figura 13.

Residuals from ARIMA $(0,1,1)(2,1,0)[12]$

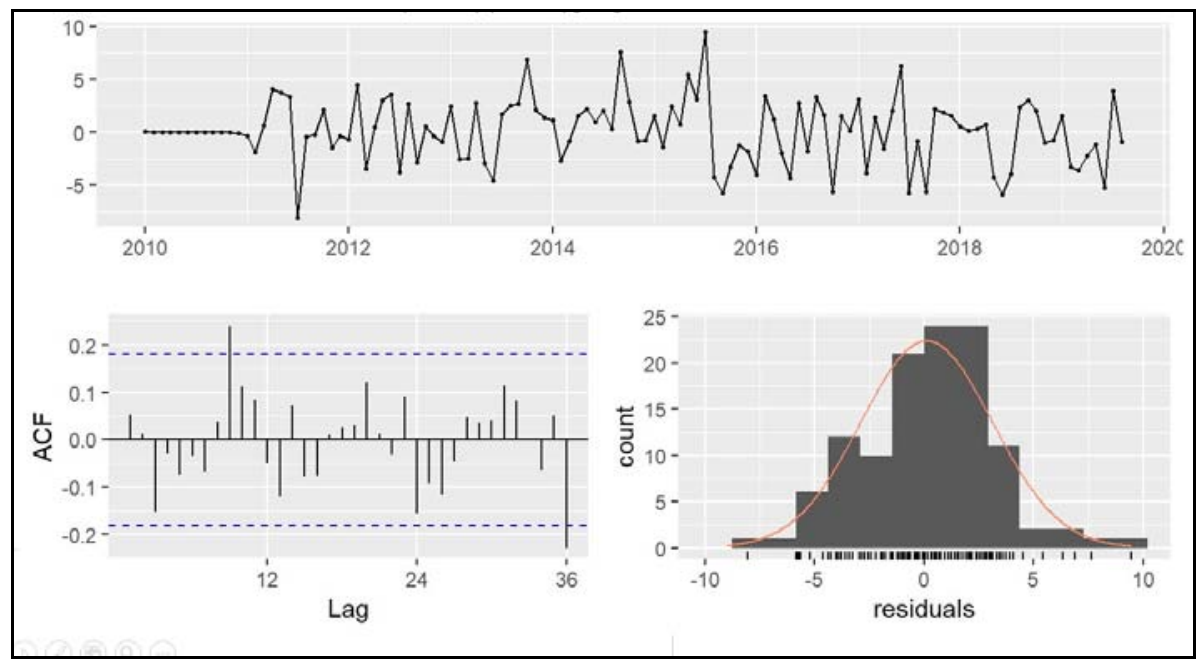

Figura 13. Residuos del modelo mod3.

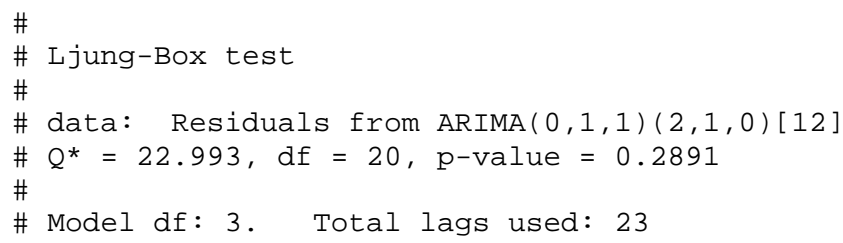

Estos residuos validan el modelo. Validado el modelo, se pasa a utilizarlo para predicción. Los valores aparecen en la siguiente tabla, y su representación gráfica en la figura 14 .

$\begin{array}{lllllll}\text { \# Point } & \text { Forecast } & \text { Lo } 80 & \text { Hi } 80 & \text { Lo } 95 & \text { Hi } 95 \\ \text { \# Sep 2019 } & 87.74220 & 83.58869 & 91.89571 & 81.38995 & 94.09445 \\ \text { \# Oct 2019 } & 80.64205 & 76.14677 & 85.13734 & 73.76711 & 87.51700 \\ \text { \# Nov 2019 } & 76.17640 & 71.36355 & 80.98925 & 68.81579 & 83.53702 \\ \text { \# Dec 2019 } & 77.55218 & 72.44146 & 82.66290 & 69.73601 & 85.36835 \\ \text { \# Jan 2020 } & 80.44323 & 75.05108 & 85.83539 & 72.19664 & 88.68982 \\ \text { \# Feb 2020 } & 2.45185 & 66.79223 & 78.11146 & 63.79621 & 81.10748 \\ \text { \# Mar 2020 } & 78.06989 & 72.15490 & 83.98489 & 69.02369 & 87.11610 \\ \text { \# Apr 2020 } & 73.35945 & 67.19965 & 79.51924 & 63.93885 & 82.78004 \\ \text { \# May 2020 } & 80.74522 & 74.34998 & 87.14045 & 70.96455 & 90.52588 \\ \text { \# Jun 2020 } & 89.70237 & 83.08007 & 96.32467 & 79.57443 & 99.83031 \\ \text { \# Jul 2020 } & 100.87338 & 94.03153 & 107.71522 & 90.40968 & 111.33707 \\ \text { \# Aug 2020 } & 99.68618 & 92.63162 & 106.74073 & 88.89717 & 110.47519\end{array}$




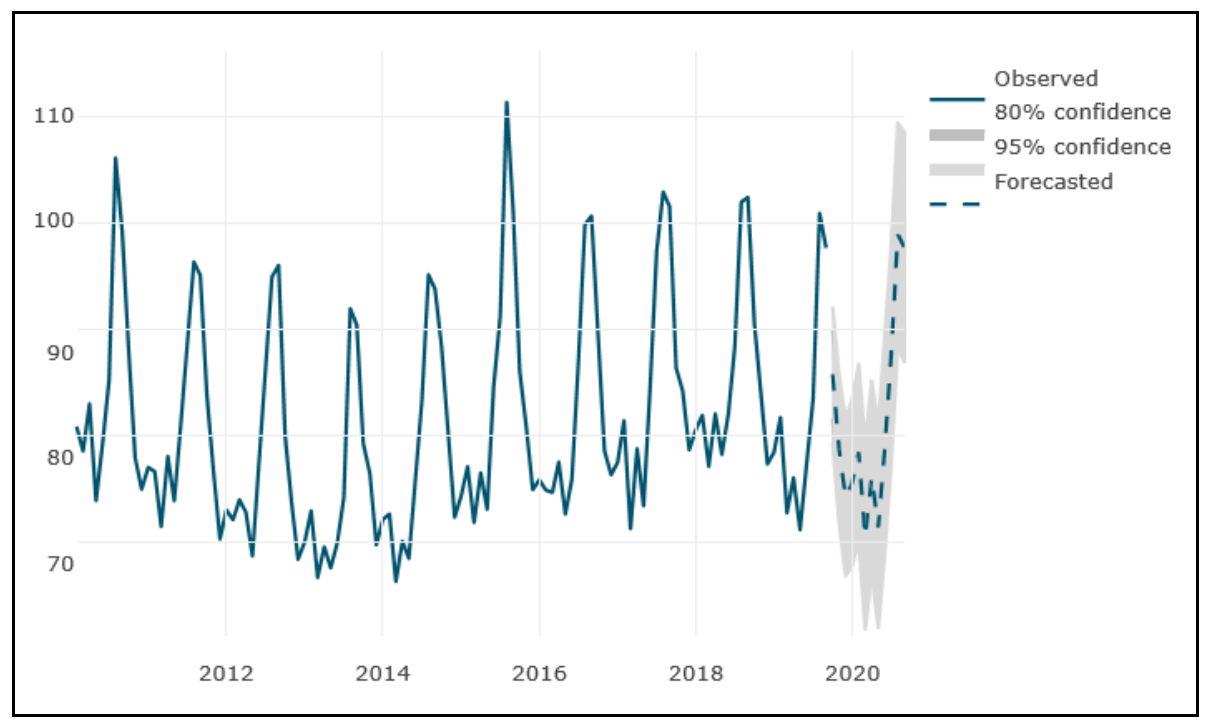

Figura 14. Predicción con el modelo mod3.

Como último paso, veamos los estadísticos del ajuste.

$\begin{array}{lllcrr}\text { \# } & \text { ME } & \text { RMSE } & \text { MAE } & \text { MPE } & \text { MAPE } \\ \text { \# Training set } & 0.1127655 & 3.009196 & 2.307399 & 0.08174433 & 2.731622 \\ \text { \# Test set } & -11.4013658 & 16.041550 & 11.401366 & -19.79214092 & 19.792141 \\ \text { \# } & \text { MASE } & \text { ACF1 } & \text { Theil's U } & \\ \text { \# Training set } & 0.5953411 & 0.05269271 & \text { NA } & \\ \text { \# Test set } & 2.9417113 & 0.77880878 & 1.764728 & \end{array}$

Si acudimos de nuevo al estadístico $M A P E$, el porcentaje de error en la submuestra train es del $2.7 \%$, y del $19.8 \%$ en la submuestra test, porcentajes que podemos considerar parecidos a los de los modelos de regresión modl y mod2. Así, pues, estos dos modelos de regresión logran predicciones comparables a las del modelo ARIMA.

\section{CONCLUSIONES}

A la vista de estos resultados parece correcto afirmar que el modelo de regresión, tan utilizado a lo largo de la historia, puede ser también utilizado para la predicción de las series temporales. 


\section{BIBLIOGRAFÍA}

- BOX, G. E. P. y JENKINS, G. M., Time Series Analysis: Forecasting and Control. San Francisco: Holden-Day, 1976.

- BOX, G. E. P.; JENKINS, G. M. y REINSEL, G. C., Time Series Analysis: Forecasting and Control, tercera edición. Englewood Cliffs: Prentice-Hall, 1994.

- CRAN, The Comprehensive R Archive Network. http://cloud.r-project.org/ (http://cloud.r-project.org/)

- HYNDMAN, R. J. y colaboradores. fpp2, programa descargable desde CRAN.

- HYNDMAN, R. J. y ATHANASOPOULOS, G., Forecasting, Principles and Practice, segunda edición. O.Texts. www.otexts.org/fpp2/, 2018.

- HYNDMAN R. J. y KHANDAKAR, Y., Automatic Time Series Forecasting: The forecast Package for R. Journal of Statistical Software, 27(2008)1-22

- KRISPIN, R., Hands-On Time Series Analysis with R., Birminghan: Packt Publishing, 2019.

- KRISPIN, R., TSstudio, programa descargable desde CRAN.

- Red Eléctrica de España, www.ree.es/es/data/publicaciones/indice-redeléctrica. 
